\title{
La importancia de incorporar el uso de Tecnologías de la Informática y las Comunicaciones TIC, en la construcción de los fundamentos integradores de la educación.
}

\section{The Importance of Incorporating the use of Information and Communication Technologies ICT, in the construction of the Integrating foundations of education.}

\author{
Héctor Rodrigo Ospina Estupiñan ${ }^{1}$ \\ ${ }^{1}$ Docente investigador, Facultad de Ciencias Económicas y Administrativas, Grupo de Investigación: Dinámicas \\ económicas y administrativas, Universidad El Bosque, Av Cra 9 \#131ª-02, Bogotá D.C, Colombia. \\ hospinae@unbosque.edu.co, hrodrigo804@gmail.com
}

Recibido: 15/ago/2020 - Revisado: 30/sep/2020

Aceptado: 30/oct/2020 - Publicado: 30/dic/2020

\begin{abstract}
Resumen En el presente siglo y en especial bajo las circunstancias acaecidas con la nueva realidad, la tecnología adquirió un papel protagónico y ha permeado todos los ámbitos del ser humano, entre ellos la educación por supuesto; en el contexto mundial, el acelerado y fuerte desarrollo e inclusión de tecnología, introdujo cambios fundamentales y relevantes en todas las esferas, especialmente en la formación; con la inclusión de tics, los procesos de enseñanza y aprendizaje se desarrollan de manera muy diferente a como se hacía en épocas pasadas, este articulo refleja por medio de un ejemplo concreto, como la incorporación de la tecnología, renueva teorías o conceptos de conocimiento tradicionales y los lleva a nuevos contextos o evoluciones para la incorporación de nuevos saberes.
\end{abstract}

Palabras claves facilitar; tics (tecnologías de la información y las comunicaciones); educación virtual; actualización.

\begin{abstract}
In this century technology acquired a leading role and has permeated all areas of human being, including education of course, in the global context the accelerated and strong development and inclusion of technology introduced fundamental and relevant changes in all spheres, especially In the formation, with the inclusion of tics, the processes of teaching and learning are developed in a very different way than was done in the past, this article reflects by means of a concrete example as the incorporation of technology renews theories or concepts of knowledge Traditional and brings them to new contexts or evolutions for the incorporation of new knowledge.
\end{abstract}

Keywords facilitate; ict (Information and Communication Technologies); education virtual update. 


\section{Introducción}

El presente artículo tiene como propósito describir por medio de ejemplos de dos instituciones de educación superior IES, de Bogotá, la manera en como la virtualización y el creciente uso de las TIC (Tecnologías de la información y las comunicaciones), se convierten en actores de primer orden, aunado al componente investigativo de la educación en el diseño y apertura de nuevas posibilidades educativas, que ayudan a mejorar las capacidades de adquisición de conocimientos de los estudiantes y a la vez son una poderosa herramienta formativa, que cada vez es más utilizada por los docentes de la educación superior.

\section{Antecedentes}

Con miras a una educación integral y de calidad que incluya el uso de las Tecnologías de la Información y la Comunicación (TIC) dentro de una sociedad globalizada, en Latinoamérica y en Colombia, se han involucrado de manera decidida en esta revolución que involucra los niveles educativos que se están dando a nivel mundial, y dado que las tecnologías ofrecen la posibilidad de renovar la modalidad pedagógica y de aprendizaje a nivel del profesorado en las aulas en cualquier nivel educativo, deben estar a la vanguardia del uso y aplicación de toda esta infinita gama de alcances y avances tecnológicos, que les sirven como herramientas para interactuar entre las diferentes asignaturas que se llevan a cabo, para que a través del uso de éstas se formen alumnos y profesores que sepan, no sólo utilizar los recursos tecnológicos, sino que también profundicen en los conocimientos adquiridos a través de estos medios, para poder ser capaces en un futuro de seguir con una educación continua a lo largo de toda su vida.

\section{Metodología}

El presente artículo de investigación, promueve el uso de la tecnología en los profesores y participantes que soportan el sistema educativo y su implementación en la educación superior, demostrado por medio de la descripción de un ejemplo aplicado en instituciones universitarias de la ciudad de Bogotá, donde el uso e incorporación de elementos tecnológicos renovó la concepción acerca del proceso de formación de estudiantes en temas específicos.

La pregunta fundamental que articula el desarrollo de este artículo se plantea así:

¿Cuál sería el impacto que debe considerar un docente de educación superior, a la hora de impartir los contenidos de su asignatura con el apoyo de las Tecnologías de la Información y la Comunicación TIC?

Objetivos

1. Establecer una reflexión holística, acerca del uso actual de la tecnología en la generación del conocimiento en la educación superior.

2. Reseñar una experiencia relacionada con el uso de la tecnología donde se pueda estimar la gestión y renovación de conocimiento, después de la integración del componente tecnológico.

3. Describir y valorar el uso efectivo de la tecnología educativa en el proceso enseñanza aprendizaje en la planeación, realización y evaluación, así como su impacto educativo institucional y social.

\section{Reflexión holística del uso de la tecnología en la educación}

En todos los entornos de aprendizaje a nivel mundial, se conjuga la tendencia de implementar procesos educativos que vayan acompañados de un mayor desarrollo y uso de las TIC como 
elementos complementarios de los procesos formativos en cualquier ámbito, ya sea escolar o laboral; a esto se le ha denominado "el surgimiento de las sociedades del conocimiento que está transformando la economía mundial y el estatus de la educación" (Namo, 1998), hace mención de la importancia de que los alumnos aprendan habilidades necesarias para enfrentarse a los cambios educativos y tecnológicos y recurran cada vez más al uso de herramientas tecnológicas para su formación; de tal manera que los padres de familia en los países desarrollados, no conciben a la educación sin cambios en el uso de las tecnologías en la enseñanza, como lo explica De La $\mathrm{Hoz}$ et al, (2019), "El sector educativo no escapa a esta realidad, incorporando las TIC como fenómeno inminente y de alta incidencia en el contexto educativo, en atención a la necesidad de promover la innovación y creatividad como mecanismo de competencia, en el que se deben enmarcar las Instituciones de Educación Superior (IES), para la operacionalización de sus funciones sustantivas".

Sin embargo, es necesario considerar dos aspectos relevantes: en primer lugar las nuevas generaciones de estudiantes quienes se sienten más atraídos por el uso y manejo de los recursos tecnológicos, por eso no es de sorprenderse como los niños fácilmente se adaptan al uso de estos elementos y la tecnología está diseñada para ser "amiga de ellos" cosa que no ocurre con generaciones anteriores que no están adaptadas al uso de estas herramientas y para adquirir el conocimiento aplicando el uso adecuado de las TIC, convirtiéndose en una combinación poderosa para el desarrollo del aprendizaje y por ende del desarrollo laboral productivo.

Por otro lado en el contexto latinoamericano se evidencian atrasos significativos en el desarrollo de los esquemas educativos, muchas veces como consecuencia de la lejanía en distancias geográficas, la carencia de elementos de comunicación y el atraso tecnológico e instruccional de nuestros modelos educativos, es por ello que las TIC, ofrecen un variado espectro de herramientas que pueden ayudar a transformar las clases actuales centradas en el profesor, aisladas del entorno y limitadas al texto de clase, en entornos de conocimiento ricos, interactivos y centrados en el alumno 0 de procesos de actualización empresarial alienados con los objetivos de desarrollo que apunten a un crecimiento sostenido y rentable de las organizaciones; (Lugo \& Ruiz, 2016) explican que el contexto del proceso, las premisas de apertura y flexibilidad para el diseño y construcción de la implementación de las políticas de uso de TIC en planes de estudios, debe fomentar la incorporación de organismos regulatorios y organizaciones que convergen en esfuerzos en pro del logro de las metas de formación y mantenimiento económico y financiero del estamento $\sin$ ir en detrimento de la calidad educativa.

Para afrontar estos desafíos con éxito, los sistemas educativos deben aprovechar las nuevas tecnologías y aplicarlas al aprendizaje, acortando distancias nos solo físicas, sino intelectuales y proporcionando a los alumnos las herramientas que les den acceso a una educación socializada, atractiva e incluyente, con el fin de que puedan a través de esquemas autorregulatorios de aprendizaje, ser mucho más eficientes en el conocimiento adquirido.

Es por ello que los gobiernos, tienen como función en este aspecto, acompañar estos esfuerzos mediante la aplicación de las reformas que modifiquen los planes de estudio enfocados al uso creciente de las TIC, como herramientas de desarrollo aplicadas a la formación.

Es fundamental que todas las personas encargadas de planear estrategias de aprendizaje en el campo educativo, se capaciten y tomen conciencia de la relevancia que implica el uso de las TIC en los procesos formativos, sin embargo para que esto se concrete, es necesario crear un marco basado en los cuatro pilares de la educación que son aprender a conocer, es decir, adquirir los instrumentos de la comprensión (capacitación permanente); aprender a hacer, para poder influir sobre el propio entorno (liderazgo y visión); aprender a vivir juntos, para participar y cooperar 
con los demás en todas las actividades humanas (contexto y cultura) y por último, aprender a ser, un proceso fundamental que recoge elementos de los tres anteriores (planeación y adaptación al cambio). (Delors J., 1996), definidos como guías del desarrollo formativo de los docentes hacia el uso de las herramientas tecnológicas aplicadas a los procesos de enseñanza, buscando articular esquemas teóricos y prácticos en pos de lograr un mejoramiento continuo de calidad, equidad $y$ pertinencia de la educación en forma universal, involucrando a los stackeholders tecnológicos, académicos y administrativos, bajo una concepción definida, clara y dinámica.

Por tal motivo, los diferentes entes gubernamentales encargados de la educación en el continente, han implementado estrategias generales de formación para los docentes mediante el diseño de trayectos formativos basados en diagnósticos pertinentes a las necesidades de cada país, lógicamente en la medida de sus capacidades así como los lineamientos determinados por la UNESCO, en el marco de la generación de una capacitación continua para profesores en las siguientes líneas de formación:

- $\quad$ Formación pedagógica.

- Formación en contenidos disciplinarios y en su enseñanza.

- Formación del uso pedagógico de las tecnologías de la información y la Comunicación.

- Formación en la organización y funcionamiento de las escuelas.

Para fines pertinentes de este documentos investigativo, la incorporación de TIC se limitará específicamente a la línea de formación del uso pedagógico de las tecnologías de la información y la comunicación, ya que son diferentes entre el modelo educativo anterior y el que se ha impuesto, debido a las circunstancias y al devenir de la formación, además de tener como base el uso de las TIC, causa mucha expectación, preocupación e inclusive miedo en muchos docentes, que tienen una formación tradicionalista y magistral y a la hora de trabajar frente a sus grupos, supone la transformación de las prácticas educativas sedentarias, por prácticas diferentes o nuevas,(SEP, 2008). El enfoque requerido será dentro del proceso de desarrollo educacional asociado con los esquemas tecnológicos y la aplicación del desarrollo pedagógico al sistema educativo de los estudiantes escolares, básicamente en la educación de nivel secundaria, sin perder el fondo que lo que se haga en educación con los jóvenes alumnos, repercutirá en el desarrollo laboral y económico de los países y las sociedades.

El objetivo fundamental de la formación del uso pedagógico de las TIC, es involucrar a los profesores con las fuentes de información digital en procesos de búsqueda sistemática, clasificación, análisis, procesamiento, producción y comunicación de esta información, con una intención pedagógica bien definida, para consolidar sus habilidades pedagógicas y digitales.

Como lo explica (Fernández et al, 2019) este es el momento oportuno para desarrollar procesos que generen dinámicas muy integradas entre el uso de las TIC y la educación, principalmente porque se cuentan con recursos digitales avanzados, una era de conocimiento interconectada, la voluntad de los entes por propiciar el uso de los recursos tecnológicos y lo mejor de todo, una generación de estudiantes que ven la tecnología como un inserto necesario para su vida, se acoplan y conviven con ella, se nutren de conocimiento e información y determinan su derrotero basados en las capacidades que esta última les ofrece.

En los últimos años, el mundo ha comenzado a vivir un proceso de cambio significativo, en todas las esferas, en este contexto, las instituciones educativas deben replantear sus propósitos para lograr individuos autónomos, optimizando sus capacidades intelectuales, que le permitan adaptarse a las constantes transformaciones de hoy. En tal sentido la educación debe ser considerada como un instrumento que permita desarrollar competencias y guiar hacia un desarrollo humano global (Coll, 2005). 
Son múltiples las razones por las cuales es preciso estudiar, comprender y aplicar el enfoque de la formación basada en competencias. Ellas son un referente de logros en los procesos formativos y no un modelo pedagógico, pues no pretenden ser una representación ideal de todo el desarrollo educativo, determinando cómo debe ser el procedimiento instructivo, el proceso desarrollador, la concepción curricular, la concepción didáctica y el tipo de estrategias didácticas a implementar. (Tobon S. 2006).

Cuando se habla de nuevas formas de aprendizaje, se analizan los cambios e innovaciones en términos de los procesos cognitivos del individuo, metodologías y modelos para promover el aprendizaje, aprovechando para ello diversos recursos y estrategias a nuestro alcance, en especial la introducción del uso de las Tecnologías de la Información y la Comunicación TIC en la educación, innovación que ha venido a ampliar y acelerar el manejo e intercambio de información y de las formas de comunicación.

Un elemento importante es el uso y la práctica de las nuevas tecnologías propuestas innovadoras para el aprendizaje, para favorecer la adquisición de conocimientos y la posibilidad de crear ambientes de aprendizaje en la educación formal, que contemplan no solamente los espacios físicos y los medios, sino también los elementos básicos del diseño instruccional, lo que logra que el estudiante, como lo explica (Moreira,2019), experimente el conocimiento de una manera versátil, reemplazando la formación tradicional, por recursos tecnológicos, que inciden y estimulan de manera positiva a adquirir el conocimiento e investigar por medio de un sinnúmero de recursos digitales y virtuales, que le da la oportunidad de acceder a bancos de información actualizados y casi que infinitos.

De tal manera que, en la actualidad, la tendencia a utilizar las TIC abre un panorama distinto creando espacios educativos y de formación de alumnos y profesores, donde el papel de este último es dar respuesta inmediata a los nuevos retos que se presentan.

\section{5 ejemplos que sirven para ilustrar la aplicación de la tecnología en la gestión del conocimiento}

Transcurría el año 2014 cuando una IES de Bogotá, D.C. recibió la renovación del registro calificado del programa de mercadeo, dentro de esa renovación se proponían modificaciones al plan de estudios, donde se establecía la incorporación y rediseño de nuevas asignaturas, varias de ellas que incorporarían el uso de tecnología como apoyo al desarrollo del programa de estudios.

En su momento la propuesta fue aceptada por el ministerio de educación, dando el aval porque la institución demostró la valía del uso de los recursos tecnológicos y el poder contar con la capacidad en talento humano como tecnología para poder llevar a cabo el proyecto.

El programa de mercadeo de la IES, se atrevió a presentar una propuesta innovadora en varios de los elementos que renovarían su propuesta académica, como la reducción del número de semestres del proceso formativo, sin afectar la carga de créditos del programa, la incorporación de asignaturas de avanzada, que llevo a la imperiosa necesidad de actualizar y capacitar a los docentes del programa en procesos de actualización y en otras circunstancias, renovar el equipo docente con profesores que pudiesen aportar el nuevo conocimiento, la integración de asignaturas con el desarrollo de proyectos conjuntos, la adopción de aulas virtuales como apoyo a la presencialidad y la renovación curricular de varias de las asignaturas, donde el viraje principal incluiría la incorporación de componentes tecnológicos, siendo asignaturas de tecnología blanda como lo es el Marketing.

Uno de los ejemplos más interesantes de este cambio se dio en la asignatura de investigación de mercados, la cual durante varios años se ministró y desarrolló de manera tradicional, es decir partiendo de la instrucción de los métodos de investigación cuantitativa y cualitativa y en donde en los últimos años los estudiantes y docentes, se decantaban en esta última, por el nivel de profundidad en reflexión 
y validación de conceptos que proponían en la investigación.

Sin embargo, el programa de mercadeo de la IES, quiso ir más allá y se decantó por incorporar la metodología de investigación correlacional.

Este método de investigación se define según (Hernández Sampieri, 2011) como, "Estudios que tienen el propósito de medir el grado de relación que exista entre dos o más conceptos o variables, miden cada una de ellas y después, cuantifican y analizan la vinculación. Tales correlaciones se sustentan en hipótesis sometidas a prueba".

Esto lo que quiere decir es que dos variables pueden estar correlacionadas y se conoce la magnitud de la asociación, se puede llegar a contar con indicios y datos para predecir con mayor o menor exactitud. Pero también se da el ejemplo en donde aparentemente dos variables estén relacionadas, pero que en realidad no sea así. A esto se le denomina Correlación Espuria, por ejemplo: imaginemos que se presenta la siguiente tendencia, a mayor estatura, mayor inteligencia; estos resultado no tendrían sentido. No podríamos decir que la estatura se correlaciona con la inteligencia, aunque los resultados del estudio así lo indicaran.

El papel fundamental de este método de investigación, radica en que tiene total pertinencia dentro del rango de la investigación social y por ende del campo de las ciencias económicas, en donde se puede establecer la relación que existe entre las variables establecidas y que a su vez se aporta información explicativa de la vinculación de cada una de ellas, es decir entre mayor sea el número de variables que estén correlacionadas en un estudio, mayor será la fuerza de la relación entre las variables y por ende la explicación de su vinculación será mucho más amplia.

Este tipo de investigación viene haciendo carrera muy fuertemente en países de Europa y Estados Unidos, principalmente por los usos estadísticos aplicados a la investigación social y empresarial para los diversos estamentos, que miden tendencias o comportamientos, para obtener información estadística de vital importancia, ya que la información que brindan es una aproximación más exacta de la percepción que tienen las personas sobre algún estimulo exógeno que lo esté afectando.

Sin embargo, para poder desarrollar este tipo de estudios, es necesario recurrir a soluciones tecnológicas que permitan realizar con mayor precisión, el cálculo de las variables y el procesamiento de datos, para ello se recurren a plataformas tecnológicas como n-vivo y el mundialmente famoso SPSS, programa estadístico informático, desarrollado por IBM, que es muy usado en las ciencias exactas, sociales y aplicadas, además de las empresas de investigación de mercado.

Para ello la IES tuvo la necesidad de hacer toda una adecuación de su estructura en el programa para adoptar la nueva tecnología y su desarrollo académico; fue necesario incorporar docentes con el perfil de investigadores estadísticos y isorpresa!, no se consiguieron muchos, se recurrió al personal extranjero de España para apoyar el proceso, luego llegó la adecuación de salas de computo, compra de licencias y capacitación de los docentes en el uso de las herramientas y la aplicación de los conceptos; el resultado fue contar hoy con un centro de investigación de mercados, que hace sus avances en la consolidación de laboratorios de investigación aplicados a la carrera, para los docentes aprender a desaprender como método de instrucción, fue necesario para romper paradigmas y superar muchas barreras de índole personal sobre el vuelco que se le dio a la asignatura.

Este aspecto llevo a establecer una reflexión interesante y fue entender las acciones específicas que conlleven a realizar actividades de innovación en la educación "... se reafirma en la propuesta de modelo formativo renovado, que ofrece al docente la posibilidad de trabajar con una gama de medios más amplia, que incluye además de los materiales impresos y de televisión, recursos informáticos.

La inclusión del uso de la computadora, materiales en audio, programas de televisión y videos de consulta, junto con la biblioteca de la IES, tienen la finalidad de actualizar y diversificar los 
materiales educativos disponibles en el aula, para crear situaciones de aprendizaje dinámicas, múltiples y variadas..." (Lerner, 2004)

Como se ha mencionado con anterioridad, darle relevancia al uso de las TIC es fundamental para que los profesores comprendan la importancia de la conexión tan estrecha que existe entre este nuevo modelo y el uso de las Tecnologías que son un puente para que los alumnos generen aprendizajes que les sean aplicables en su vida futura $y$ complementen un perfil de egreso integral.

Al revisar los aprendizajes del ejemplo relacionado, se encontró que, al abrirnos a nuevas opciones de generación del conocimiento, se busca generar en los profesores un estímulo, para hacer que sus clases sean realmente didácticas, variadas y enriquecedoras, donde encontrarían una gran cantidad de ventajas que les ofrecen el uso de las herramientas tecnológicas aplicadas a la educación, tales como:

- Incorporación de las TIC en el proceso de aprendizaje del alumno.

- Acceso a la gran cantidad de información que ofrece Internet.

- Medios de comunicación efectivos y económicos.

- Aumentar el nivel de escolaridad de los entornos educativos y laborales.

- Al ser las TIC un medio motivador y dinámico, contribuye a aumentar el aprovechamiento de los alumnos.

- Se logrará que los alumnos sean capaces de entender el medio natural que los rodea y les permita generar acciones que ayuden al mantenimiento y cuidado de la biodiversidad.

\subsection{Otra experiencia de incorporación de TIC, motivada por la nueva realidad}

Marzo de 2020, el mundo es azotado por la pandemia del Covid 19, todo lo que se conoce como estatus quo del hombre y la sociedad, queda revalidado en un abrir y cerrar de ojos, todo el mundo debe confinarse, las palabras aislamiento, mascarilla, protectores, guantes, desinfectar y demás, se vuelven el glosario de términos más comunes, el mundo debe encerrarse en sus casas y todo se ve apocalíptico, pero en esta ocasión a este fenómeno de las pandemias, que siempre han acompañado la historia de la humanidad, existe un factor diferenciador que determina una nueva realidad y es que la vida y la cotidianidad del hombre se vuelven virtuales.

Un proceso que se sabe tenía que desarrollarse paulatinamente, se termina imponiendo en todas $y$ cada una de las acciones que realiza el ser humano para continuar su existencia, el trabajo, las diligencias, el día a día, se enmarca en el contexto de las aplicaciones de las páginas web, los domicilios y la fuerte influencia del e-commerce como factor dependiente del mantener una rutina de vida determinada.

La educación no se excluye de este contexto y, al contrario, se ve impulsada a adoptar nuevas medidas y formas para incorporar rápidamente a la población a los procesos educativos, buscando lograr dos preceptos fundamentales, universalidad de la educación y cobertura a la población.

En este orden de ideas una IES como todas las que cohabitan en la mega urbe de Bogotá, la cual está especializada en el campo de las ciencias de la salud y donde los contenidos de formación estaban muy ligados a los modelos tradicionales de la instrucción, donde la tecnología y la virtualidad, eran consideradas un complemento y apoyo al desarrollo de los planes de estudio, decide embarcarse en una apuesta bastante incierta y es migrar a un esquema donde se garantice una educación con calidad y conservando estándares iguales o similares a lo que se requiere para desarrollar a cabalidad los contenidos temáticos, con la dificultad que eso implica.

Cuando se hizo el balance de los resultados del primer año de gestión, los resultados fueron impresionantes, $90 \%$ de retención de estudiantes, niveles de desempeño sostenidos, nivel de matrícula de nuevos estudiantes, con metas cumplidas al $100 \%$ en el $70 \%$ de los programas y con mínimos hasta del $80 \%$ en los restantes, 
¿Cómo se logró esto? ¿Dónde estuvo la diferencia y por qué obtuvo mejores resultados que muchas otras IES en donde el panorama es mucho más sombrío?

La respuesta está precisamente en ese anterior soporte tecnológico que coadyuvaba a la formación tradicional, en donde se venían implementando paulatinamente el desarrollo de aulas virtuales, mediante el uso de plataformas LMS, el desarrollo de actividades diferenciales al formato tradicional de desarrollo de trabajos, como el uso de formatos de video, presentaciones interactivas y demás aspectos; entonces los pedagogos y los actores tecnológicos, cuando llegó la nueva situación de encierro, se enfocaron en afinar el proceso, enmarcado en algo que se denominó - Estrategia de Continuidad Académica- la cual se definió en los siguientes principios:

- La nueva realidad llegó para quedarse y es la institución quien debe adaptarse para garantizar el normal desarrollo de los planes de estudios.

- Se debe facilitar el acceso a la educación a los estudiantes, sin afectar su formación y brindándole nuevos espacios de interacción.

- Asegurar los recursos técnicos, tecnológicos y pedagógicos que permitan el logro del propósito.

- Establecer metodologías dinámicas que mediante el uso de la tecnología y modelo de construcción de conocimiento desde la comunidad, asegure generar las competencias formativas requeridas en los diversos perfiles.

Para lograr esto fue necesario toda una transformación basada en lo que se tenía, trabajando en actualizar rápidamente a los docentes y estudiantes en el manejo de las aulas virtuales, definiendo una suite de trabajo muy sencilla y fácil de operar para todos, recurriendo al uso de diversas aplicaciones de comunicación libre como webwex, google meet o teams, desarrollo de actividades que involucren el uso de tecnología para la construcción de las evidencias de aprendizaje, videos, paneles, aplicaciones etc. y la incorporación de la gamificación como un elemento fundamental en este proceso.
Esto se convirtió en la base de un modelo de formación adaptado y con resultados comprobados en el logro del proceso educativo de los estudiantes y generando el desarrollo de nuevas habilidades y capacidades mediante el uso de la incorporación tecnológica y el acercamiento de la pedagogía a técnicas de despliegue digitales y virtuales, que permiten mejorar la cobertura y el alcance de la educación.

\subsection{Hallazgos del análisis de las dos experiencias desde el contexto de la investigación.}

Bajo este marco de referencia y observando estos dos casos de éxito, se observa la importancia de incorporar componentes formativos y tecnológicos y afinar procesos como la investigación educativa a coadyuvar, a sentar las bases para encausar los recursos humanos y económicos a que haya lugar y aprovechar las garantías que ofrecen las autoridades educativas y empresariales, para la capacitación del personal docente y de enseñanza, con la finalidad de tener un impacto en la mejora de la vida social del alumno y el trabajador.

Un proceso de investigación de esta índole siempre será viable, dado que es un problema actual y tangible al que nos enfrentamos los profesores de educación superior y son tres factores visibles que se pueden mencionar los cuales son la causante de la problemática:

1. Los profesores y capacitadores empresariales de las nuevas generaciones provienen de una formación universitaria y adolecen de conocimientos pedagógicos básicos relacionados con los desarrollos tecnológicos, lo cual genera un desconcierto en el uso específicamente pedagógico de las TIC.

2. Los profesores que sí cuentan con una formación normalista, han sido preparados de acuerdo a los planes y programas de estudio de los años 80's y 90's, épocas en las que ni siquiera se vislumbraba la importancia que 
tomarían las tecnologías que en aquel entonces empezaban a nacer y que en un futuro no muy lejano serían utilizadas como herramientas de gran poder dentro de la pedagogía.

3. Y el último grupo de profesores que no tienen los estudios normalistas, que llevan muchos años de servicio, y que como están a punto de jubilarse no tienen la intención de intentar aprender a utilizar las TIC, prefiriendo seguir con su método de trabajo tradicionalista hasta el día en que se retiren.

Por las observaciones anteriores, es necesario involucrar el componente investigativo de la educación, como perspectiva que ayude a comprender algunos de los problemas ya identificados, que pueden ser subsanados con un esfuerzo conjunto por parte de todos los involucrados en el proceso de enseñanza.

\subsection{Investigación educativa y los beneficios de la incorporación del uso de la tecnología.}

Tanto en el campo educativo, como en el resto de las ciencias, la investigación se ha constituido en una actividad precisa y elemental. Por este motivo, se ha originado la investigación educativa, como disciplina que "trata las cuestiones y problemas relativos a la naturaleza, epistemología, metodología, fines y objetivos en el marco de la búsqueda progresiva de conocimiento en el ámbito educativo." (Arnal J. Rincón D, 1994)

El concepto de investigación educativa se ha ido modificando a medida que han surgido nuevos enfoques para el tratamiento de los fenómenos educativos. Actualmente, son variados los significados atribuidos a la expresión Investigación Educativa, dependiendo de la diversidad de objetivos y características que se le establecen. Según varios expertos, esta temática contribuye a abordar el siguiente apartado vinculado con los paradigmas en el estudio de los sucesos educativos y su relación con la problemática que genera la búsqueda del conocimiento.
Según (Bungue 1986) "Cuando hablamos de trasfondo científico, nos referimos al cuerpo de conocimientos preexistente, el cual está compuesto por conceptos teóricos, datos, técnicas, procedimientos, generalizaciones empíricas, supuestos, etc." Entonces se debe tener en consideración que los problemas no surgen de la nada, toda teoría determina los problemas que se pueden formular como científicos, o de cuestión filosófica y esto claramente permite reafirmar el postulado de Bunge donde expresa claramente que "El proceso creador de la ciencia arranca del reconocimiento de problemas y culmina con la construcción de teorías, donde los problemas se convierten en la materia prima para generar las investigaciones y el nivel de las mismas es el resultado de la magnitud del problema.

En este orden de ideas es importante determinar que los esquemas de investigación en la educación, centran sus esfuerzos en resolver los problemas que se refieren al cómo y de qué manera lograr un mejor acceso al dominio de conocimiento, no como foco total del desarrollo investigativo pero si como uno de los vectores que determinan las vías de la investigación y es parte del punto que ha generado un debate en torno a los métodos de investigación en las ciencias sociales, especialmente en el campo de la educación, el cual lleva ya mucho tiempo en el ámbito del contexto educativo a todo nivel. Sin duda, uno de los asuntos centrales de la investigación educativa reside en la ausencia de una metodología unánimemente reconocida por los expertos como la más adecuada para su investigación. (Maceri, 2007). Cierto es que la pretensión de unanimidad es demasiado ambiciosa, pero sí, en cambio, podemos intentar dilucidar qué metodología de la investigación educativa es preferible a otra.

Otro componente que es fundamental dentro del proceso del desarrollo de la investigación educativa, son los paradigmas que surgen como elementos generadores de polémica en cuanto a su valides en la aplicación de los conceptos orientados a la investigación. Según (Kuhn, 1975) los paradigmas son realizaciones científicas 
universalmente reconocidas que durante cierto tiempo proporcionan modelos de problemas y soluciones a una comunidad científica. Cada comunidad científica comparte un mismo paradigma y cuando lo replantea entra en crisis y es cuando se da la revolución científica.

La literatura más reciente sobre investigación educativa coincide en señalar sólo tres paradigmas fundamentales: positivista, interpretativo y críticoreflexivo. Además de estos tres paradigmas, últimamente se menciona el paradigma emergente o praxis sustentado por diversos autores, entre ellos (Hernández, R., Fernández, C. \& Baptista, P. (2006). El paradigma emergente, aún en proceso de construcción es una posibilidad de integración con características propias y que determina que siempre existirá una tercera opción o posibilidad de concepto frente a un desarrollo o conclusión de conocimiento. (Maceri 2007).

\section{TIC en educación superior}

La educación superior al igual que todos los niveles de la formación académica deben responder a los requerimientos del entorno global, dentro de los cuales uno de primera línea es el adecuado manejo de la tecnología que deben adquirir los estudiantes y por otra parte la inclusión pedagógica de la misma, los procesos formativos por parte de las instituciones y los docentes, no como un nuevo modelo educativo, no como la panacea para el aprendizaje, sino más bien como un nuevo elemento que potencializa nuevas formas de aprendizaje, acordes a los cambios del entorno externo en general (Moreno,2008).

Puede decirse que la tecnología enriquece los procesos de educación, en tanto plantea una aproximación al conocimiento y la información mediante exploración activa, interactiva y didáctica, lo cual motiva altamente a los estudiantes de este nivel por los mismos procesos de desarrollo cognitivo en el que se encuentran. Las TIC también facilitan a los jóvenes un papel autorregulador de su aprendizaje, en cuanto a tiempos, ritmos y estilos, lo cual les acerca de forma mucho más amena al aprendizaje significativo, sin el rol del docente vigilante y autoritario, sino más bien como orientador, obviamente continuando este con su rol formativo clave en los procesos de enseñanza y aprendizaje del nivel básico sin ser "reemplazados" por la virtualidad, en cambio docentes y estudiantes avanzan hacia nuevas formas de enseñar $y$ aprender con mayor dinamismo que con los solos modelos tradicionales.

Sin embargo, es fundamental recalcar que por más que se conciba la tecnología dentro de la educación superior como elemento fundamental del contexto actual para la enseñanza, poco o nada podrá lograrse con su inclusión en este nivel formativo, si de una parte no responde a un modelo pedagógico completo para su inclusión y por otro lado se requiere una participación más activa del estado, con políticas y recursos para garantizar unos mínimos que permitan instaurar una educación básica donde promuevan, apoyen y fortalezcan la inclusión de TIC en la enseñanza a todo nivel y con óptimos niveles de cobertura. A manera de ejemplo, en este sentido el Plan Decenal de Educación de Colombia plantea unos aspectos que deben tenerse en cuenta en la inclusión de TIC en educación básica, válidos para el contexto latinoamericano así:

1. Infraestructura tecnológica y conectividad. Es indispensable que tanto el gobierno como las mismas entidades de educación básica, sean de carácter público o privado, deben movilizarse así a la consecución de infraestructura tecnológica y conectividad, pues ello constituye el sustrato físico mínimo para que las TIC puedan incluirse eficientemente en la formación básica.

2. Cualificación en la formación docente. Como lo retomaremos más adelante, hoy es un hecho que los estudiantes de educación superior están adelantados frente al docente en lo que al conocimiento y uso de la tecnología se refiere, por tal razón, es necesario que tanto las instituciones educativas como los mismos docentes, estén constantemente en formación y 
cualificación en lo que a la inclusión de TIC en educación se refiere.

3. Implementación de estrategias didácticas que a través de TIC faciliten el aprendizaje autónomo, colaborativo y pensamiento crítico. Dicha implementación se logra mediante la reestructuración de currículo, del modelo pedagógico y el conocimiento y apropiación de didácticas específicas apoyadas principalmente por herramientas Web 2.0.

4. Revisión del sistema de evaluación. El sistema de evaluación de una institución educativa de formación básica no puede ser el mismo después de la inclusión de TIC, ya que este solo hecho conlleva otras necesidades y formas de concebir e implementar la evaluación.

La inclusión de TIC en educación básica es especialmente útil para los niños que conforman esta población, ya que ellos son nativos digitales, es decir nacieron en el mundo de la tecnología ya inserta en los diferentes contextos, por esta razón para ellos es natural y muy intuitivo el uso de dicha tecnología, por esta facilidad que tienen en el uso de la, el que la encuentren incluida en sus procesos formativos hace que se motiven altamente a la hora de aprender, además que pueden también profundizar en casa sobre los mismos temas que trabajan en las instituciones educativas.

Las TIC se convierten en un medio para que los estudiantes desarrollen nuevas habilidades como capacidad del desarrollo de multitareas, manejo de información mundial rápida, interacción con otros a través de los medios virtuales, el uso de juegos on line como apoyo a las asignaturas de los currículos de formación básica, (PRONAP. 2007).

Otro de los puntos que debemos plantearnos desde la inclusión de TIC en la educación básica, es que en este momento los estudiantes (nativos digitales) de dicho nivel saben más de TIC y del manejo tecnológico en general que los docentes (inmigrantes digitales) y dicha realidad obliga a que las instituciones educativas de formación básica replanteen el currículo y los modelos pedagógicos, ya no como productos terminados sino como procesos vivos en constante construcción que permitan que estos cambios sean tenidos en cuenta y que en ese sentido los docentes se cualifiquen de la manera y al ritmo adecuado para responder a los nuevos estudiantes y sus necesidades.

"Estos estudiantes poseen una asombrosa capacidad de adaptación en toda actividad que implique las TIC en general y en particular la computadora en Internet. Capacidad para enfrentar y resolver problemas a partir de una habilidad para la comunicación interactiva y simbólica, independientemente de su capacidad verbal; todo ello por el lenguaje de signos propios de la programación cibernética que permite un entendimiento común" (Córica y Dinerstein. 2009).

"Dada la naturaleza integrativa, visual, auditiva y kinestésica de las TIC y de sus múltiples aplicaciones, son predominantemente activos, visuales, propensos al intercambio $y$ emprendedores mediante su empleo". (Córica y Dinerstein. 2009).

Es por ello que es posible determinar que la innovación pedagógica en combinación con el apoyo tecnológico en la continuidad de los procesos formativos, determinado bien sea por las circunstancias evolutivas de la sociedad 0 acelerados por cambios repentinos del modo vida, como ha sido esta horrible pandemia, son el punto de partida de un nuevo camino que hay que recorrer para reenfocar el accionar de los diversos stackholders de la educación, como lo explica (Moreno y Correa, 2020) "Posiblemente estemos ante la generación de un nuevo paradigma educativo. Lo anterior podría llevarnos a pensar que la tragedia de una pandemia puede dejarnos aprendizajes y situaciones que nos llevan a ver realmente la causa de los problemas que aquejan a nuestra sociedad y a nuestros discentes.

\section{Conclusiones}

Como conclusiones se pueden establecer aspectos como que el ser humano transforma, adapta y crea contenidos de aprendizaje de acuerdo a sus necesidades, deseos o 
requerimientos de conocimiento, por lo anterior los nuevos ambientes de aprendizaje, que incorporan apoyos tecnológicos y uso de recursos digitales, deben ofrecer a los docentes y alumnos la capacidad de hacer un uso adecuado de la tecnología, con el fin de acceder al conocimiento, poniendo a su disposición los medios que le permitan desarrollar aplicaciones de sus habilidades académicas, para dar solución a los problemas o toma de decisiones de su día a día.

Las prácticas educativas tradicionales ya no les aportan a los estudiantes ni a los docentes todas las habilidades necesarias para la supervivencia económica en el sitio de trabajo de hoy. La Cruz Alcoer (2006) refuerza este concepto por medio de la identificación de problemas derivados de la insuficiente dotación económica a los centros de formación y la manera como se incorporan a los profesores a los medios virtuales como parte de su quehacer docente. Sería conveniente que los que tienen responsabilidad en la formación del profesorado en nuevas tecnologías intentasen detectar cuales son las causas por las cuales el profesorado activo se resiste a implicar profesionalmente y a aplicar estos nuevos medios en su actividad docente.

De igual manera los estudiantes deberían aplicar estrategias para resolver problemas y usar las herramientas apropiadas para aprender, colaborar y comunicarse.

Tanto actores tecnológicos como docentes deben comprender que hoy día el enfoque de la educación está basado en lineamientos participativos, donde la comunicación es elemento fundamental de este proceso, más aun cuando los desarrollos comunicacionales van totalmente acompañados de los avances tecnológicos, la evolución de nuestra sociedad está basada precisamente, en el desarrollo de redes y contextos socialmente activos, donde las barreras geográficas e idiomáticas quedan totalmente desvirtuadas gracias al devenir tecnológico, en este aspecto la enseñanza retoma bases y principios fundamentales para incorporar nuevos elementos formativos atractivos y sofisticados que cautivan a los estudiantes, amigos y aliados del contexto tecnológico, aunado a la formación.

Además resulta fundamental compartir las metas de aprendizaje, desarrollar actividades colaborativas y atender y dar seguimiento específico a todos los estudiantes, para responder a sus necesidades a través de educación permanente y accesible para todos, por medio de sistemas de comunicación eficientes y si esto se transfiere al contexto virtual, se puede determinar que el concepto de educación con apoyo tecnológico o virtual desde la óptica de la comunicación, según Villar (2004), la herramienta comunicativa y pedagógica, la capacidad de docente de dominar el conocimiento por medio de la aplicación tecnológica y la correcta ministración del conocimiento a sus estudiantes, aunada a una plataforma en la cual se desarrolla la vida académica, es lo que se denomina apoyo tecnológico a presencialidad académica, factor fundamental para el desarrollo del conocimiento actual.

Hay que considerar que la situación en la que nos encontramos, plantea un escenario donde la humanidad está inmersa en un proceso de devastación de las recursos, desintegración social y de valores y aprecio por el prójimo, increíblemente la pandemia ha hecho que nos fijemos un poco más en nosotros y los demás, que pensemos en el bienestar colectivo y el autocuidado, la naturaleza también aprovecha esta pausa para oxigenarse y reconstruirse, para el ser humano esto puede ser una ventaja y en esto la educación es un factor clave, empezando por que quienes nos dedicamos a la docencia, fuimos obligados a salir de nuestra de zona de confort, actualizarnos y evolucionar a la par de las tendencias tecnológicas, incorporando nuevas acciones y generando estrategias de formación idóneas y adaptativas a la nueva realidad.

Agradecimientos. Expreso mis sinceros agradecimientos a la Fundación Universitaria Tecnologico Comfenalco, por permitirme desarrollar mi segunda publicación en su revista Teknos, en 
segundo lugar, agradezco a la Dra Luz Bety Diaz, compañera, inspiración, ejemplo y gran esposa, así como a la Dra. Marha Obregón y el Dr. Antonio Alonso González, por apoyar y facilitar el camino, en el logro de mis metas y propósitos.

\section{Referencias}

Arnal, J.,Rincón D. (1994), Latorre A.: "Investigación educativa. Fundamentos y metodologías". Edit. Labor, Barcelona.

Bunge, Mario. 1986. "La investigación científica. Su estrategia y su filosofía". Ariel, Barcelona.

Coll, C. (2005). Las Competencias en la educación escolar: Algo más que una moda y mucho menos que un remedio. Aula de innovación educativa Núm. 161. México. pp. 34-55.

Còrica J , Dinerstein P. 2009. Diseño Curricular y Nuevas Generaciones. Editorial Virtual Argentina. $1^{a} \mathrm{Ed}$ Mendoza, Argentina.

De-la-Hoz-Franco, Emiro, Martínez-Palmera, Olga, Combita-Niño, Harold, \& Hernández-Palma, Hugo. (2019). Las Tecnologías de la Información y la Comunicación y su Influencia en la Transformación de la Educación Superior en Colombia para Impulso de la Economía Global. Información tecnológica, 30(1), 255-

262. https://dx.doi.org/10.4067/S0718-

07642019000100255

Delors J. (Comp) (1996). Los cuatro pilares de la educación. En: la educación encierra un tesoro. pp. 89-103. México. UNESCO

Farfan, M., Reyes,I., (2017). Gestión educativa estratégica y gestión escolar del proceso de enseñanza-aprendizaje: una aproximación conceptual, Análisis de Problemas Universitarios, vol. 28, núm. 73, 2017, Universidad Autónoma Metropolitana

Fernández, E., Ordoñez, E., Morales., Cevallos, J. (2019) La competencia digital en la docencia universitaria Octaedro Ediciones ISBN: 978-84-17667-44-3

Hernández, R., Fernández, C. \& Baptista, P. (2006). Recolección y análisis de los datos cualitativos. Metodología de la investigación (4a. ed., pp. 597604). México: McGraw-Hill.

Hernández Sampieri. (2011). Metodología de la Investigación. 11 ava edición, Mexíco, Mc Graw Hill

Kuhn, T. (1975). La estructura de las revoluciones científicas. Fondo de Cultura Económica. México.

Lacruz, Alcoer, Miguel. (2006). Nn.tt. Y cambio curricular. Universidad de Castilla La Mancha. Escuela Universitaria de Magisterio de Ciudad Real. C/ Hidalgos, $n^{\circ} .1,5^{\circ}$. 1. C.P. 13004- CIUDAD REAL.

Lerner, L. (2004). Leer y escribir en la escuela. El papel del conocimiento didáctico en la formación del maestro. Fondo de cultura económica. México. pp. 165-190.

Lugo.M, Ruíz. V, (2016) Revisión comparativa de iniciativas nacionales de aprendizaje móvil en América Latina: los casos de Colombia, Costa Rica, Perú y Uruguay. Unesco doc, digital library ISBN: 978-92-3-100145-1

Maceri, S. (2007). Hacia una metodología de la investigación educativa. Madrid.

MEN, (2006). Ministerio de Educación Nacional de Colombia, planes y programas de la educación media en Colombia.

Moreno C. M. (2008) Innovación social y educativa. La educación en ambientes virtuales como alternativa innovadora. México. ILCE.

Moreno-Correa SM. La innovación educativa en los tiempos del Coronavirus. Salutem Scientia Spiritus 2020; 6(1):14-26. Universidad Javeriana.

Moreira, P. (2019). El aprendizaje significativo y su rol en el desarrollo social y cognitivo de los adolescentes. Rehuso, 4(2), 1-12. Recuperado de: https://revistas.utm.edu.ec/index.php/Rehu so/article/view/1845

Namo, de M. G. (1998). Nuevas propuestas para la gestión educativa. Modernización, telecomunicación y conocimiento: la quinta ola en gestión escolar. Biblioteca para la actualización del maestro. México. pp. 1108-110.

Plan Nacional Decenal de Educación 2006-2016 Colombia.

http://www.plandecenal.edu.cohttp://basic a.sep.gob.mx/seb2008/web/html/archivos/ RNacional/miercoles 12dediciembre/Taller experienciaEnciclomediaSalonGpdf

PRONAP. (2007). Sugerencias metodológicas para el fortalecimiento de las estrategias de aprendizaje en los estudiantes de educación secundaria y telesecundaria. Cursos estatales de actualización. Dirección de educación superior, departamento de actualización. México.

SEP, (2008). Los nuevos materiales educativos. Un 
Héctor Rodrigo, O. E. (2020). La importancia de incorporar el uso de Tecnologías de la Informática y las Comunicaciones TIC, en la construcción de los fundamentos integradores de la educación. Teknos Revista Científica, 2020(2), 55-68.

acercamiento al modelo renovado de

Telesecundaria. pp. 38-45. México.

Tobón, S. (2006) Aspectos básicos de la información basada en competencias. Talca. Proyecto Mesesup. Bogotá: ECOE.

Villar Gabriela (2004). Las nuevas tecnologías en la Educación superior universitaria. Universidad Virtual de Quilmes, Argentina cap 327 pags 